\title{
ANALYSIS OF WALL ANTIGENS OF ASPERGILLUS FUMIGATUS BY TWO-DIMENSIONAL IMMUNOELECTROPHORESIS
}

\author{
Veronica M. Hearn and D. W. R. Mackenzie \\ Mycological Reference Laboratory. London School of Hygiene and Tropical Medicine, \\ Keppel Street, London WC1E 7 HT
}

\section{Plates VI-XII}

\begin{abstract}
SummaRY. Water-soluble and surface-located antigens from Aspergillus fumigatus mycelium were analysed by two-dimensional electrophoresis (2D IEP) with selected sera. A complex pattern was seen when a water-soluble fraction reacted with hyperimmune sera. Significantly fewer precipitin peaks were seen when wall-located antigens were used in the 2D IEP system; this made possible the detection of a small number of recurring antigen-antibody interactions when sera from patients with aspergilloma or allergic bronchopulmonary aspergillosis were analysed. Many of these recurring precipitates were affected by lectins with specificity for $\alpha$-D-glucose, $\alpha$-D-mannose or $N$-acetyl-D-glucosamine. All the antigens were susceptible to hydrolysis by pronase, confirming the glycoprotein composition of many of these macromolecules.
\end{abstract}

\section{INTRODUCTION}

The mycelium of Aspergillus is a useful source of antigens for the serological investigation of bronchopulmonary aspergillosis in man (Wada, 1960; Azuma et al., 1967; Biguet et al., 1967; Bardana et al., 1972; Kim et al., 1978; Hearn and Mackenzie, 1979; Hearn et al., 1980b). Although salt fractionation of the water-soluble extract of mycelium yields a range of useful antigens (Hearn et al., 1980b) and wall antigens have been shown to be reactive in several in-vitro systems (Hearn and Mackenzie, 1979, 1980) there are problems with batch variability, and diagnostic serology requires standardised reproducible reagents. The present paper describes a study of wall-located antigens that were prepared from Aspergillus fumigatus mycelium by extraction with the non-ionic detergent Triton X-100, and the water-soluble antigenic components, used in routine tests in this laboratory, by two-dimensional immunoelectrophoresis (2D IEP) which is useful for the analysis of complex antigenic mixtures (Huppert et al., 1978; Longbottom, 1978).

\section{MATERIALS AND METHODS}

Preparation of water-soluble antigens from whole mycelium. A water-soluble (WS) extract was prepared from 3-day-old mycelium of $A$. fumigatus (either strain no. NCPF2109 or 
NCPF2140) by physical rupture of the organism, separation of the soluble constituents from the insoluble residue by centrifugation and concentration by freeze-drying (Hearn et al., 1980b).

Preparation of water-soluble extract from cell wall. The insoluble residue, obtained during the preparation of the WS antigens, was washed repeatedly in hot water $\left(50^{\circ}-60^{\circ} \mathrm{C}\right)$ until the washings were clear. The pellet was then extracted, as a $10 \%(\mathrm{w} / \mathrm{v})$ suspension in $0.05 \mathrm{M}$ $\mathrm{NH}_{4} \mathrm{HCO}_{3}$, at $p \mathrm{H} 8.0$ and $30^{\circ} \mathrm{C}$ for two 2-h periods, with stirring. The combined supernates, after centrifugation, were dialysed and concentrated approximately 30 -fold against $0.9 \% \mathrm{NaCl}$ in $10 \%(\mathrm{w} / \mathrm{v})$ polyethylene glycol (PEG) 6000 and the insoluble residue removed by centrifugation. The supernatant bicarbonate extract $(\mathrm{BE})$ was stored at $-20^{\circ} \mathrm{C}$.

Preparation of wall antigens. The insoluble residue, after bicarbonate extraction, was treated with $0.5 \%(\mathrm{v} / \mathrm{v})$ Triton X-100 (scintillation grade from Koch-Light, Colnbrook, Bucks.) in 0.05M $\mathrm{NH}_{4} \mathrm{HCO}_{3}$ at $p \mathrm{H} 8.0$ and $30^{\circ} \mathrm{C}$ for two 2-h periods, with moderate stirring (Hearn and Mackenzie, 1979). The combined supernates (TE) were then treated in the same way as the BE preparation. Concentration of the antigenic extract leads to a concomitant concentration of the detergent.

Fractionation of wall antigens. Triton X-100 was partially removed from the TE extract by overnight adsorbtion at $4^{\circ} \mathrm{C}$ on to Bio-Beads SM-2 (Bio-Rad, Richmond, Ca, USA) at a protein level of $5 \mathrm{mg} / \mathrm{g}$ of beads and a final detergent concentration of $1 \%(\mathrm{v} / \mathrm{v})$, according to Holloway (1973). Removal of the residual triton was by disruption of the micelles with an ethanol-ethylene glycol mixture followed by ultrafiltration (Frasch, 1976). The filtrate was monitored for the presence of triton by measuring the absorption at $276 \mathrm{~nm}$. The retained material, after dialysis and concentration against PEG 6000, served as a source of WS/TE antigens (Hearn and Mackenzie, 1980). All wall fractions were stored at $-20^{\circ} \mathrm{C}$.

Production of antisera. Antiserum was obtained by hyperimmunisation of New Zealand White rabbits with either the WS fraction from 3-day-old mycelium of $A$. fumigatus strain 2109 or 2140 (Hearn et al., 1980b); it was used unconcentrated.

Antiserum was raised in rabbits against the culture-filtrate antigens of $A$. fumigatus. This serum was a gift from Dr K. Holmberg of the Statens Bakteriologie Laboratorium, Stockholm; it was used unconcentrated.

Human serum samples were obtained from patients suffering from allergic bronchopulmonary aspergillosis (ABPA) (10 specimens) or aspergilloma (15). Some had been submitted to this laboratory for serological examination while the others were kindly given by Dr J. Edwards, MRC Pneumoconiosis Unit, Llandough Hospital, Penarth, S. Glamorgan.

Concentration of patients' sera. The bulk of the plasma proteins was precipitated with caprylic acid (Steinbuch and Audran, 1969). The IgG component and a portion of the IgA, which remained in the supernate, were concentrated fivefold against PEG 6000 . This procedure was done on selected sera from patients with ABPA.

Two-dimensional immunoelectrophoresis was done on slides covered with a 1.2-mm layer of tris-barbitone buffered agarose gel at $p \mathrm{H} \mathrm{8.6} \mathrm{(Bøg-Hansen,} \mathrm{Brogren} \mathrm{and} \mathrm{McMurrough,} \mathrm{1974).}$ In addition to the antigen, a reference albumin/anti-albumin system was included in each test. Antigens were used routinely at the following protein concentrations: WS, BE and TE fractions at $0.14 \mathrm{mg}$ per test and the WS/TE fraction at $0.18 \mathrm{mg}$ per test. The sera were used at the following concentrations: anti-albumin at $2 \mu \mathrm{l} / \mathrm{cm}^{2}$; homologous and culture-filtrate antisera at 8 $\mu \mathrm{L} / \mathrm{cm}^{2}$; sera from patients with aspergilloma at $12 \mu \mathrm{l} / \mathrm{cm}^{2}$; sera from patients with ABPA (concentrated fivefold) at $16 \mu \mathrm{L} / \mathrm{cm}^{2}$. Antigen-antibody precipitates were designated peaks 1,2 , 3 etc., beginning with the peak of highest anodic mobility. $R f$ values were measured relative to albumin. Peaks giving the same Rf values were given the same peak number. Other details of this procedure are reported elsewhere (Hearn et al., 1980b).

Lectin-binding experiments. All lectins were used at a concentration of $1 \mathrm{mg}(\mathrm{dry} \mathrm{wt}) / \mathrm{ml}$ of intermediate gel solution. Concanavalin A (Con. A), A grade, was from Cal-biochem, San Diego, Ca, USA; Triticum vulgaris lectin (wheat germ agglutinin, WGA), lectins from Ricinus communis (castor-bean type I and type II) with specificities for $\mathrm{N}$-acetyl-D-glucosamine, $\mathrm{N}$-acetyl-D-galactosamine and D-galactose, respectively, were from Sigma Chemicals, Poole, Dorset.

Proteolytic hydrolysis. Pronase (Koch-Light) was used at a final concentration of $29 \mathrm{mg} / \mathrm{ml}$ of gel solution at $p \mathrm{H} \mathrm{8.6.} \mathrm{The} \mathrm{separated} \mathrm{antigens} \mathrm{were} \mathrm{electrophoresed} \mathrm{in} \mathrm{the} \mathrm{second} \mathrm{dimension}$ 
overnight through the pronase contained in the intermediate gel. In these experiments the albumin was run in the second dimension only; it was applied in a well placed immediately above the intermediate gel.

Chemical analyses. The method of Dubois et al. (1956) was used to estimate total neutral sugars. Protein was measured with the Folin-Ciocalteau reagent (Lowry et al., 1951) in the presence of a final triton concentration not greater than $0 \cdot 1 \%$.

\section{RESULTS}

Reactivity of water-soluble (WS) and wall (TE) antigens towards antiserum raised against culture-filtrate antigens

Initially, the WS and TE antigens were analysed by IEP against the serum raised against culture filtrate antigens. The number of precipitation peaks ranged from approximately 20 in the WS fraction to $7-8$ constituents in the TE and in the WS/TE fractions which gave similar precipitation profiles (figs. 1a and $b$ ).

\section{Reactivity of WS and TE antigens towards mycelial and human sera}

When the WS and TE fractions were tested against a serum that was homologous to the WS fraction, the WS fraction showed 12-15 components while the TE extract showed the presence of approximately eight components. The two most intensely stained peaks in the two fractions, and some weaker ones, fused on tandem electrophoresis but some well defined peaks were found to be peculiar to one or other of the extracts (fig. 2a). When tested against a pool of sera from patients with aspergilloma, the two major precipitates in each fraction fused in a reaction of identity on tandem electrophoresis (fig. 2b).

\section{Analysis of wall material}

Analysis of the BE material showed a protein : carbohydrate ratio $(\mathrm{P}: \mathrm{C})$ of approximately $1.5: 1$; the yield per $\mathrm{g}$ (wet weight) of mycelium was $0.45 \mathrm{mg}$ of protein, i.e., $0.075 \%$ recovery, calculated on the basis of total protein and carbohydrate. Subsequent elution with bicarbonate containing Triton X-100 yielded material with a protein content of $0.75 \mathrm{mg} / \mathrm{g}$ wet weight of mycelium extracted and a $\mathrm{P}: \mathrm{C}$ ratio of approximately $1 \cdot 7: 1$ (i.e., a yield of $0 \cdot 13 \%$ ). Further fractionation of the TE extract, as described in the methods section, gave a WS/TE fraction. This material had a $\mathrm{P}: \mathrm{C}$ ratio of $1: 1$ and an average yield of $0.02 \%$. The TE and WS/TE extracts were analysed by 2D IEP against an individual serum sample from a patient with aspergilloma and were then run in tandem to detect common immunological constituents. One of the two most prominent peaks in the TE preparation showed immunological identity with the single, major peak in the WS/TE fraction (fig. 3a). When the latter was run in tandem with the BE extract, the two well defined peaks with relatively high anodal mobility were found to be peculiar to the $\mathrm{BE}$ preparation 
while the smaller, more intensely stained peak, midway between the anode and the cathode was present only in the WS/TE fraction (fig. 3b).

\section{Reactivity of wall antigens from strain 2109 towards patients' sera}

The TE and WS/TE fractions were tested for precipitation reactivity against sera from patients with ABPA or aspergilloma. The 15 individual serum samples from patients with aspergilloma all reacted positively in 2D IEP with the TE extract. The minimum number of peaks from any one serum was two (seen with $13 \%$ of the sera), while the greatest number of peaks from any one serum was $12 ; 80 \%$ of the sera had five peaks or more. For ease of classification the peaks were arbitrarily separated into three groups on the basis of their mobility relative to the albumin marker. Antigens with $\mathrm{Rf}$ values of $0.70-0.90$ (anodal antigens, containing four constituents as a maximum) were designated group $\mathrm{A}$; those with $\mathrm{Rf}$ values of $0.30-0.50$ (median antigens, again with a maximum of four components) were designated group $\mathrm{B}$; and those with $\mathrm{Rf}$ values of $0.05-0.20$ (cathodal antigens, constituting a group of, at most, four components) were designated group C.

All sera tested contained antibodies to at least two group-B antigens. Twelve sera contained antibodies to group-A antigens, almost invariably peaks 1 and 2; peaks 3 and 4 were detectable in very few samples and were characterised by their pale colour and low antibody titre. Only seven of 15 serum samples contained detectable antibodies to group-C antigens (fig. 4a).

\section{WS/TE antigen reactivity}

The reaction of WS/TE antigens with test sera from seven aspergilloma patients was characterised by the presence of one predominating precipitation peak in the B-group position. As many as 10 precipitates could be detected with an individual serum but these components, in general, tended to be weakly reacting. Group-A antigens were detectable with five of the seven sera tested; these reactions were almost invariably weak, showing very faint colour and low antibody titre. Whether or not they were the same antigens as those seen in the TE fraction is not yet known. Group-C antigens were seen with only two of the seven sera analysed (fig. 4b).

\section{$T E$ antigen reactivity with $A B P A$ sera}

- With the 10 individual ABPA sera tested, precipitation profiles were very similar to those seen when the same antigenic mixture was allowed to react with aspergilloma sera. However, the reactions were weaker and $280 \mu \mathrm{L}$ of a fivefold concentration of these sera were needed to produce a profile comparable with that produced by $200 \mu \mathrm{L}$ of unmodified serum from patients with aspergilloma. All sera gave peaks 1 and 2 from antigen group A; all sera contained three or four components from group-B antigens; group-C antigens reacted with serum from four of 10 cases (fig. 5a). 


\section{WALL ANTIGENS OF ASPERGILLIUS}
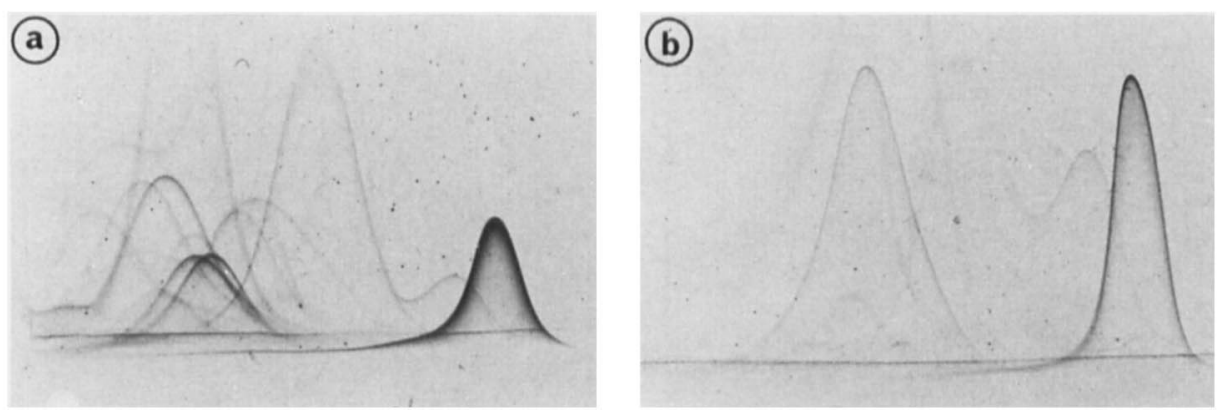

FIG. 1.-Mycelial extracts of Aspergillus fumigatus strain 2109 tested by two-dimensional immunoelectrophoresis (2D IEP) against a serum raised against culture-filtrate antigens. (a) WS fraction; (b) TE fraction. See Materials and methods for definition of fractions.
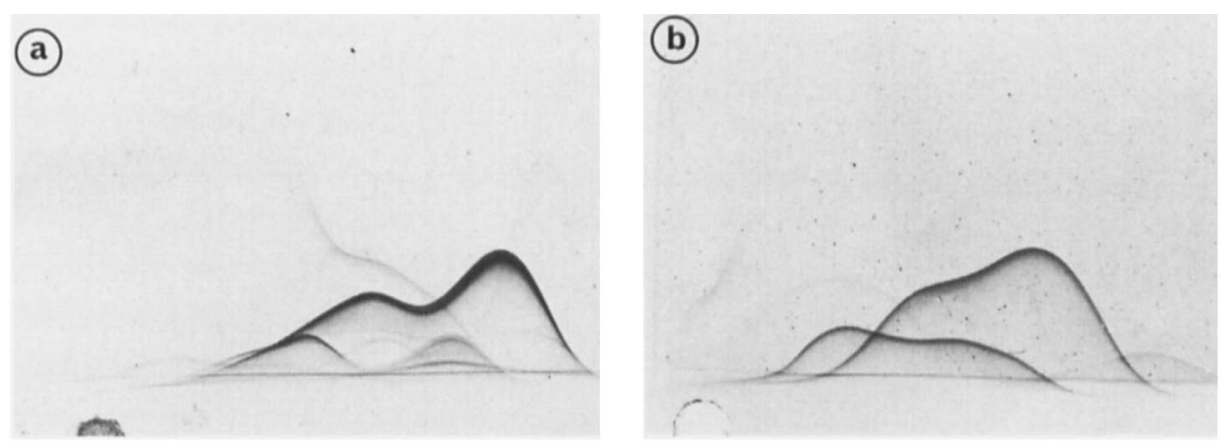

FIG. 2.-Tandem immunoelectrophoresis of the mycelial extracts of $A$. fumigatus strain 2109 . (a) The WS and TE fractions tested against a homologous antiserum; (b) the same fractions tested against a pool of selected sera from patients with aspergilloma. 
WALL ANTIGENS OF ASPERGILLUS
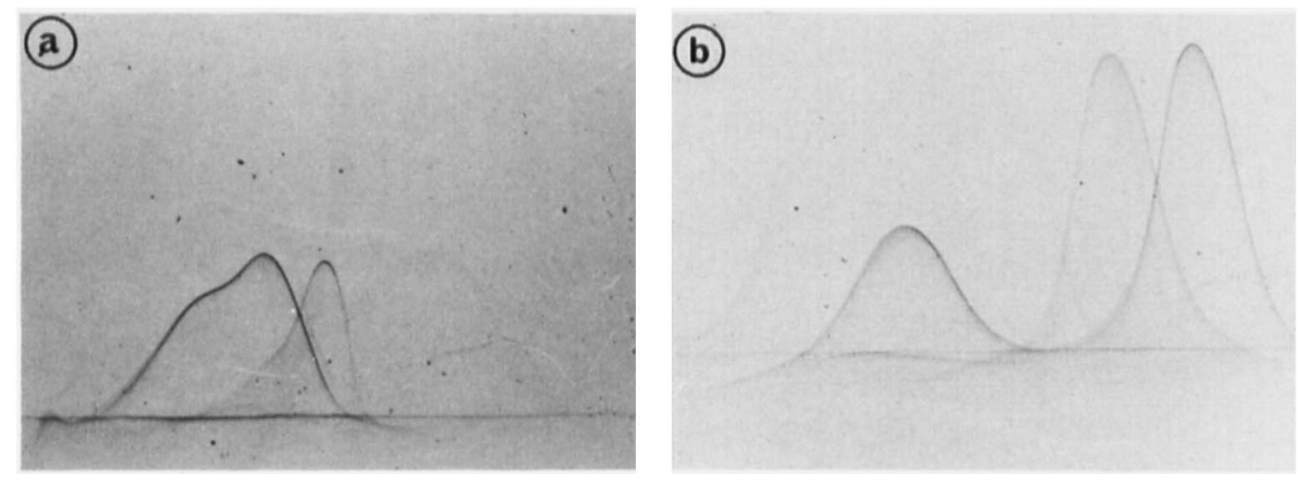

FIG. 3.-Tandem immunoelectrophoresis of the wall antigens of $A$. fumigatus strain 2109 . (a) TE (in the leading well) and WS/TE reacted with a serum from an individual patient with aspergilloma; (b) BE (in the leading well) and WS/TE reacted with the same serum.
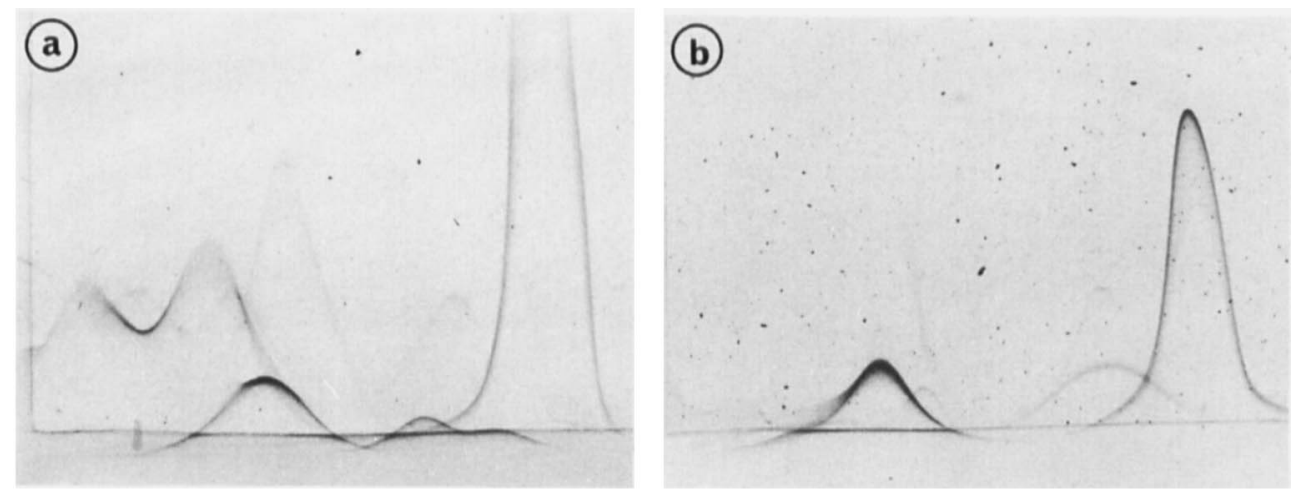

Fig. 4.-Wall antigens from mycelium of $A$. fumigatus strain 2109 tested by 2D IEP against sera from individual patients with aspergilloma. (a) TE with serum no. 5314; (b) WS/TE with serum no. 2632. 
WALL ANTIGENS OF ASPERGILLUS
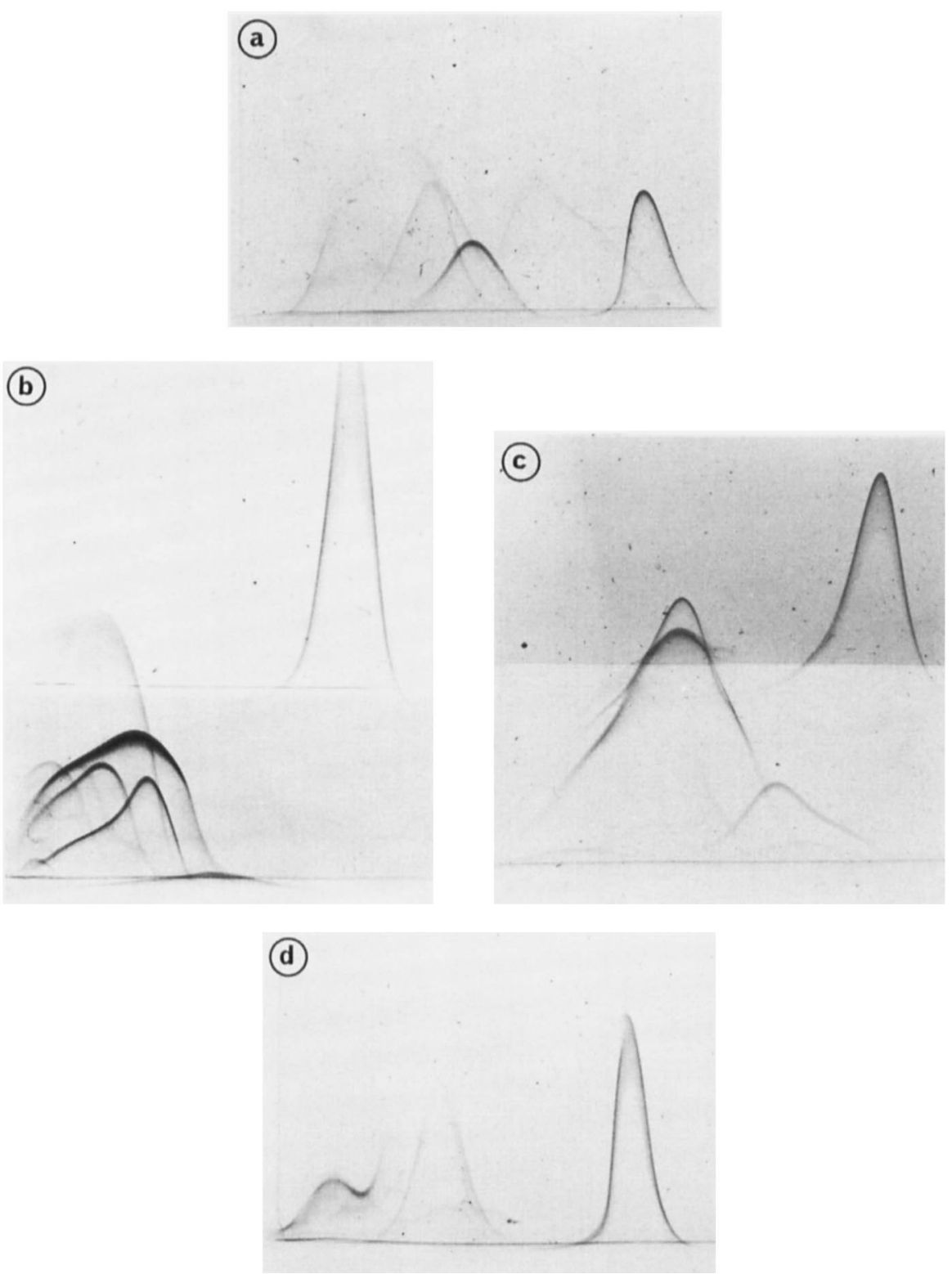

Fig. 5.-Wall antigens from mycelium of A. fumigatus strain 2109 tested against sera from patients with allergic bronchopulmonary aspergillosis $(\mathrm{ABPA})$ (concentrated $\times 5$ ) or aspergilloma. (a) TE analysed against a selected pool of sera from ABPA patients; (b) intermediate-gel IEP with sera from two different patients with aspergilloma in the lower and upper gels tested against a TE fraction; (c) intermediate-gel IEP with a selected pool of ABPA sera in the lower gel and serum from a patient with aspergilloma in the upper reference gel tested against a TE fraction; (d) a WS/TE fraction analysed against a selected pool of ABPA sera. 
Intermediate gel electrophoresis was used to determine whether different serum specimens contained non-identical antibodies to the antigens in these extracts. Fig. 5b shows that with two different aspergilloma sera in the intermediate and reference gels, all precipitation occurred in the lower gel. The top of one component is to be seen in the upper gel but this simply indicates a low antibody titre, not non-identity because the line is continuous. Similarly, when a concentrated serum from a patient with ABPA was placed in either the intermediate or upper gel and compared with that from a patient with aspergilloma, all precipitation again occurred in the lower gel (fig. 5c).

\section{WS/TE antigen reactivity with $A B P A$ sera}

All the five individually concentrated sera had antibodies detectable by two or three antigens from group B. Two of the five sera responded to group-A antigens; the same proportion responded to group-C antigens (fig. 5d).

\section{Wall antigens from strain 2140}

To compare the two strains of $A$. fumigatus in routine use in this laboratory, wall antigens were also prepared from strain 2140 . The TE fraction contained antigens of all three groups which reacted with serum antibodies from aspergilloma patients. In all cases, the group-B antigens predominated; there was a significant group-C response while, in general, reactivity with $\mathrm{A}$ antigens was weak. With the same test serum, a TE preparation from strain 2140 gave a profile closely related to that obtained with strain 2109 (fig. 6a).

With two of the five sera from patients with aspergilloma tested against a WS/TE fraction from strain 2140 , only group-B components were detectable. Only one of five contained group-C antigens while three of five contained group-A antigens. Again, this fraction from the two strains was compared against the same test serum (fig. $6 \mathrm{~b}$ ). The results are summarised in table I.

Tandem electrophoresis of the TE fractions from the two strains against a selected pool of sera from patients with aspergilloma showed immunological fusion of the major, group-B, antigenic constituents. Similarly, tandem electrophoresis of the two WS/TE fractions showed identity of the major and the minor antigenic moieties. A comparison of the reactivity of the wall antigens, prepared from the two strains of $A$. fumigatus mycelium, was made on a few test sera (table II).

\section{Lectin-binding experiments}

Con. A, with specificity towards $\alpha$-D-glucose and $\alpha$-D-mannose residues, and WGA, with specificity towards $\mathrm{N}$-acetyl-D-glucosamine, were the two lectins that had a measurable effect on the 2D IEP profiles of all the antigens studied. Castor-bean lectins type I and II were without demonstrable effect, under the conditions used. In a WS extract, Con. A interacted with many components. The four most anodal constituents and also some of the weakly 


\section{TABLE I}

Two-dimensional electrophoresis of wall antigens from two strains of Aspergillus fumigatus with sera from patients with aspergilloma or allergic bronchopulmonary aspergillosis

\begin{tabular}{|c|c|c|c|c|c|c|c|c|}
\hline \multirow{2}{*}{$\begin{array}{c}\text { Aspergillus } \\
\text { strain no. and } \\
\text { antigen fraction* }\end{array}$} & \multirow{2}{*}{$\begin{array}{l}\text { Number of } \\
\text { aspergilloma } \\
\text { sera tested }\end{array}$} & \multicolumn{3}{|c|}{$\begin{array}{c}\text { Number }(\%) \text { of sera precipitating } \\
\text { with one or more } \\
\text { antigens of group }\end{array}$} & \multirow{2}{*}{$\begin{array}{l}\text { Number of } \\
\text { ABPA } \\
\text { sera tested }\end{array}$} & \multicolumn{3}{|c|}{$\begin{array}{l}\text { Number }(\%) \text { sera precipitating } \\
\text { with one or more } \\
\text { antigens of group }\end{array}$} \\
\hline & & A & B & $\mathrm{C}$ & & A & B & $\mathrm{C}$ \\
\hline $\begin{array}{l}2109 \mathrm{TE} \\
2109 \mathrm{WS} / \mathrm{TE} \\
2140 \mathrm{TE} \\
2140 \mathrm{WS} / \mathrm{TE}\end{array}$ & $\begin{array}{r}15 \\
7 \\
5 \\
5\end{array}$ & $\begin{array}{l}12(80) \\
5(71) \\
5(100) \\
3(60)\end{array}$ & $\begin{array}{l}15(100) \\
7(100) \\
5(100) \\
5(100)\end{array}$ & $\begin{array}{l}7(47) \\
2(29) \\
5(100) \\
1(20)\end{array}$ & $\begin{array}{r}10 \\
5 \\
0 \\
0\end{array}$ & $\begin{array}{c}10(100) \\
2(40) \\
\ldots \\
\ldots\end{array}$ & $\begin{array}{c}10(100) \\
5(100) \\
\ldots \\
\ldots\end{array}$ & $\begin{array}{c}4(40) \\
2(40) \\
\ldots \\
\ldots\end{array}$ \\
\hline
\end{tabular}

ABPA = Allergic bronchopulmonary aspergillosis; * see Materials and methods for definition of fractions. 


\section{WALL ANTIGENS OF ASPERGILLUS}
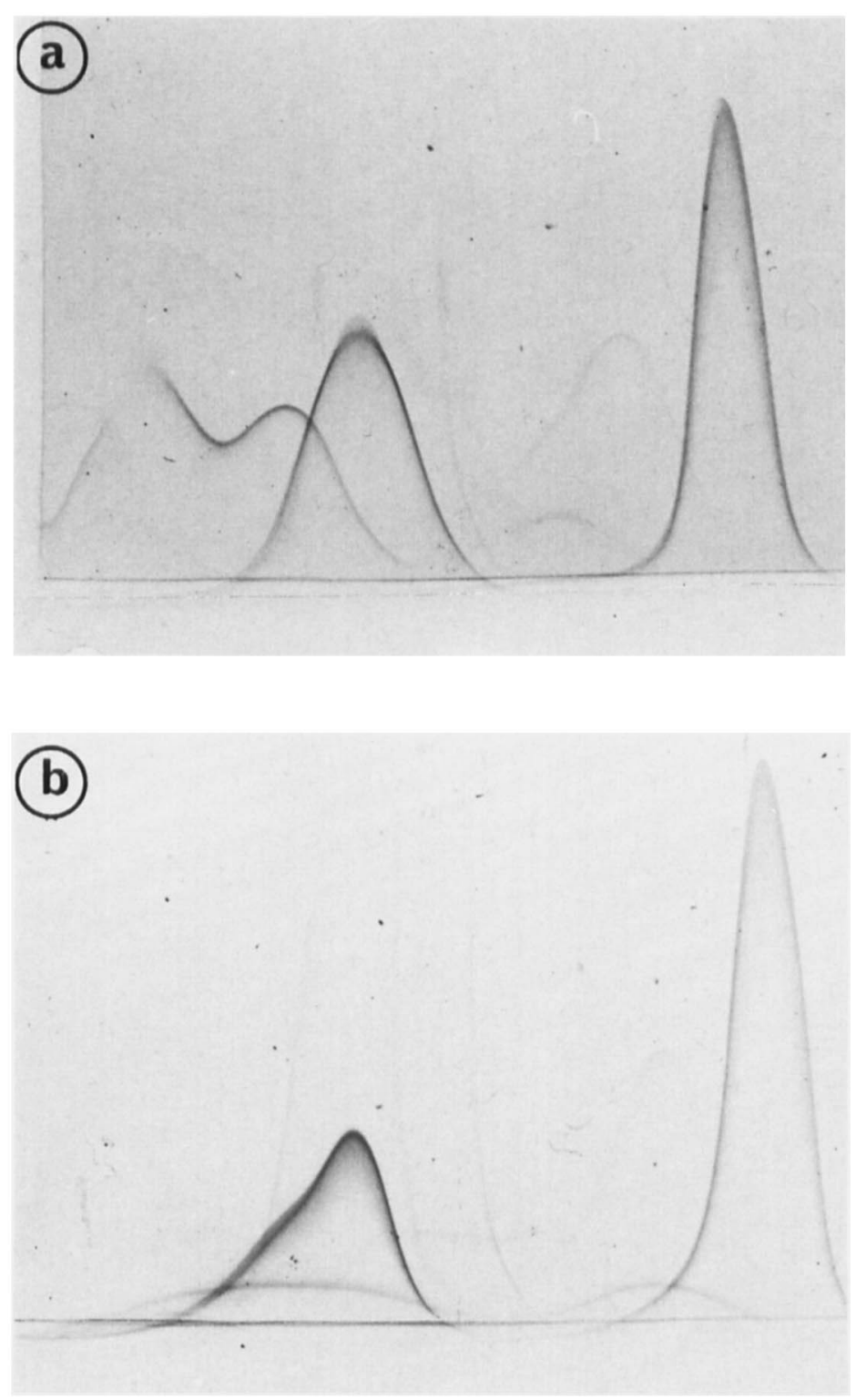

FIG. 6.-Wall antigens from the mycelium of $A$. fumigatus strain 2140 tested against sera from individual patients with aspergilloma. (a) TE with serum no. 5314; (b) WS/TE with serum no. 2632. 
TABLE II

Tandem electrophoresis of wall antigens from two strains of Aspergillus fumigatus with sera from patients with aspergilloma

\begin{tabular}{|c|c|c|c|c|c|c|}
\hline \multirow[b]{2}{*}{ Serum no. } & \multicolumn{2}{|c|}{$\begin{array}{l}\text { Number of peaks with } \\
\text { antigen fraction TE* } \\
\text { from strain }\end{array}$} & \multirow{2}{*}{$\begin{array}{l}\text { Number of } \\
\text { shared } \dagger \\
\text { peaks }\end{array}$} & \multicolumn{2}{|c|}{$\begin{array}{c}\text { Number of peaks with } \\
\text { antigen fraction WS/TE* } \\
\text { from strain }\end{array}$} & \multirow{2}{*}{$\begin{array}{l}\text { Number o } \\
\text { shared } \\
\text { peaks }\end{array}$} \\
\hline & 2109 & 2140 & & 2109 & 2140 & \\
\hline $\begin{array}{r}2632 \\
592 \\
5314 \\
1276 \\
4137\end{array}$ & $\begin{array}{c}8 \\
6 \\
12 \\
6 \\
\mathrm{NT}\end{array}$ & $\begin{array}{r}9 \\
11 \\
10 \\
10 \\
\text { NT }\end{array}$ & $\begin{array}{c}6 \\
4 \\
6 \\
4 \\
\ldots\end{array}$ & $\begin{array}{c}6 \\
\text { NT } \\
10 \\
\text { NT } \\
2\end{array}$ & $\begin{array}{c}8 \\
\text { NT } \\
2 \\
\text { NT } \\
2\end{array}$ & $\begin{array}{l}4 \\
1 \\
2\end{array}$ \\
\hline
\end{tabular}

reacting group-B antigens could still be seen. WGA, on the other hand, interacted with the group-A antigens, leaving group B apparently unaffected (figs. $7 a-c$ ).

A TE extract with a precipitin profile showing approximately six constituents against a homologous antiserum lost all but one anodal peak when Con. A was incorporated into an intermediate gel. With WGA in the intermediate gel a different, weakly reacting, anodal peak was absorbed while two of the group-B antigens were somewhat retarded in the intermediate gel (figs. 8a-c). The only two components visible when a WS/TE fraction was reacted against a homologous antiserum were lost on incorporation of Con. A in the intermediate gel.

\section{Pronase hydrolysis}

Incorporation of pronase in the intermediate gel led to the loss of the previously discernible precipitins when the WS and TE extracts were tested against human serum (figs. 9a and $b$ ). When homologous serum was used, traces of some group-A precipitates remained after electrophoresis of the WS preparation through pronase; with a TE extract, traces of some group-B precipitates remained.

\section{Discussion}

Mycelial extracts of $A$. fumigatus showed the presence of many antigenic components when analysed by 2D IEP. Shared antigens found in the mycelium and secreted into the culture medium were demonstrated by positive reactivity between the structural antigens and an antiserum raised against culture filtrate antigens (cf. Biguet et al., 1967; Yokota et al., 1977). Tandem electrophoresis showed the presence of common antigens in the WS and wall fractions with the latter demonstrating fewer precipitates against all sera 
tested. Wall antigens were therefore selected for further study because their relatively simple precipitation profiles could be more easily analysed.

When the TE and WS/TE fractions were tested against sera from patients with aspergilloma, a distinct pattern emerged. These antigens gave only a few sharp, intensely stained precipitates of low peak height, which are a measure of high avidity and high antibody titre (Axelsen, Bock and Krøll, 1973; Longbottom, 1978). By the technique of intermediate-gel IEP it was established that these preparations detected similar antibodies in serum samples from different patients with either aspergilloma or ABPA though the levels of specific IgG antibodies in the allergic patients were very low. Only macromolecules of high affinity are detectable by this technique and the possibility therefore exists that weaker antigen-antibody systems were lost (Bøg-Hansen, Bjerrum and Ramlau, 1975).

Many of the detectable antigens have a glycoprotein structure (cf. Hearn and Mackenzie, 1979); many interacted with Con. A, showing the presence of $\alpha$-D-glucose or $\alpha$-D-mannose residues or both, while essentially all reactivity was abolished by pronase hydrolysis. Some antigens interacted with WGA, showing the presence of $\mathrm{N}$-acetyl-D-glucosamine residues. Partial retardation by WGA of some peaks, which were lost with Con. A, presumably indicates the presence of neutral and glucosamine residues on these macromolecules. No interaction was found with the castor-bean lectins, specific for D-galactose and $\mathrm{N}$-acetyl-D-galactosamine. Stoddart and Herbertson (1978) showed the presence of galactosamine residues on the surface of $A$. fumigatus walls using fluorescein-labelled soybean lectin. The TE extract contains little galactosamine (Hearn and Mackenzie, 1979) which suggests that this detergent fails to solubilise galactosamine-containing structures that are present in crude wall preparations; this fact may account for these divergent findings.

Yields of $0.2 \%$ (TE) and $0.02 \%$ (WS/TE) are the average obtainable from the two strains of $A$. fumigatus (wet weight of mycelium) and are low when compared with the WS fraction which varies from $1 \%$ to $2 \%$. However, in in-vitro serological systems the wall antigens show 3-8 times the sensitivity of the WS preparation (Hearn, Proctor and Mackenzie, 1980a). It is of interest that Salvin and Ribi (1955) in their studies on Histoplasma capsulatum found the complement-fixing activity of a cell wall fraction was far greater than that protoplasm. Batch variability of wall antigens has been shown both chemically and immunologically (Hearn et al., 1980a). However, all batches of TE and WS/TE contain one or more of the B-reactive group of antigens, though the level varies. Some of these antigens may be found in the WS fraction after hyphal breakage but due to the complexity and variability of this system (Hearn et al., 1980b), positive identification is difficult. Detergent action was required to release the major antigenic constituents of TE and WS/TE from crude wall material; they were absent from a BE extract (cf. Pine, Boone and McLaughlin (1966) who found that prolonged sonic treatment was required to release antigens from the cell walls of Histoplasma capsulatum).

Whether any of these antigens possess chymotryptic activity remains to be established (Biguet et al., 1967). The recurrence of some antigen-antibody 


\section{WALL ANTIGENS OF ASPERGILLUS}
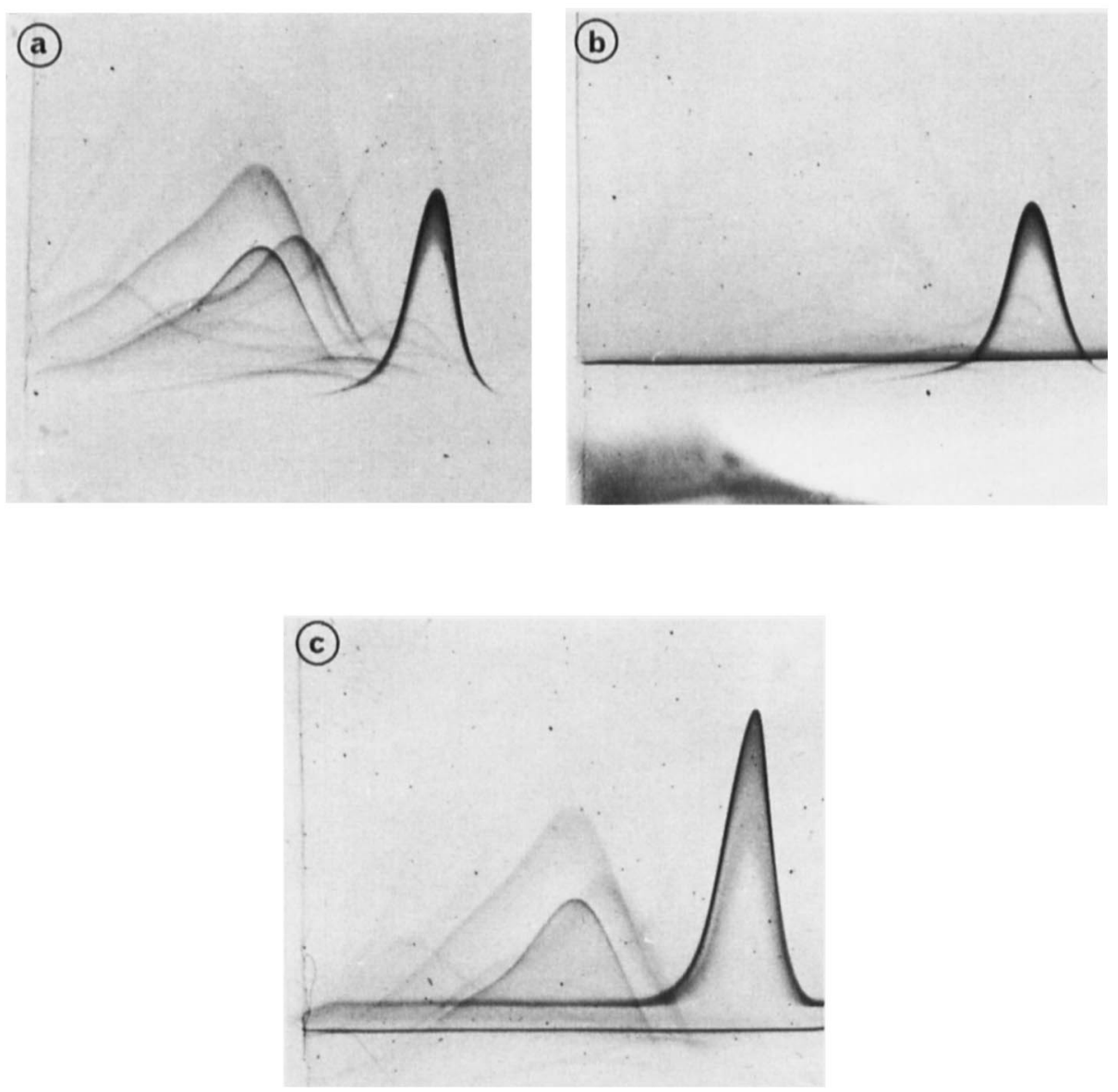

FIG. 7.-Intermediate-gel IEP of the WS fraction from $A$. fumigatus strain 2140 tested against a homologous antiserum. (a) No lectin in the intermediate gel; (b) Con. A in the intermediate gel; (c) WGA in the intermediate gel. 


\section{WALL ANTIGENS OF ASPERGILLUS}
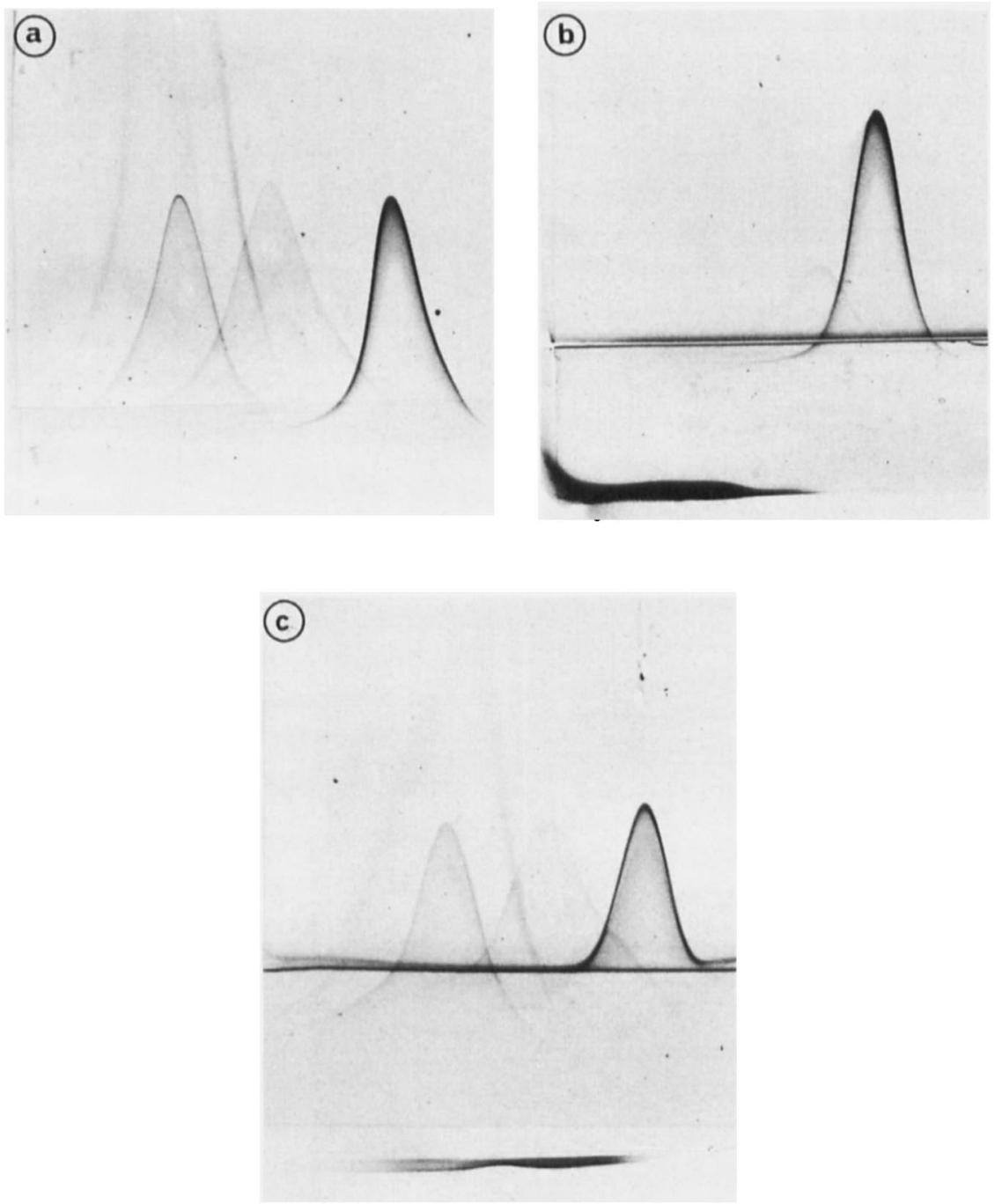

FIG. 8. - Intermediate gel IEP of the TE fraction from A. fumigatus strain 2140 tested against a homologous antiserum. (a) No lectin in the intermediate gel; (b) Con. A in the intermediate gel; (c) WGA in the intermediate gel. 


\section{WALL ANTIGENS OF ASPERGILLUS}
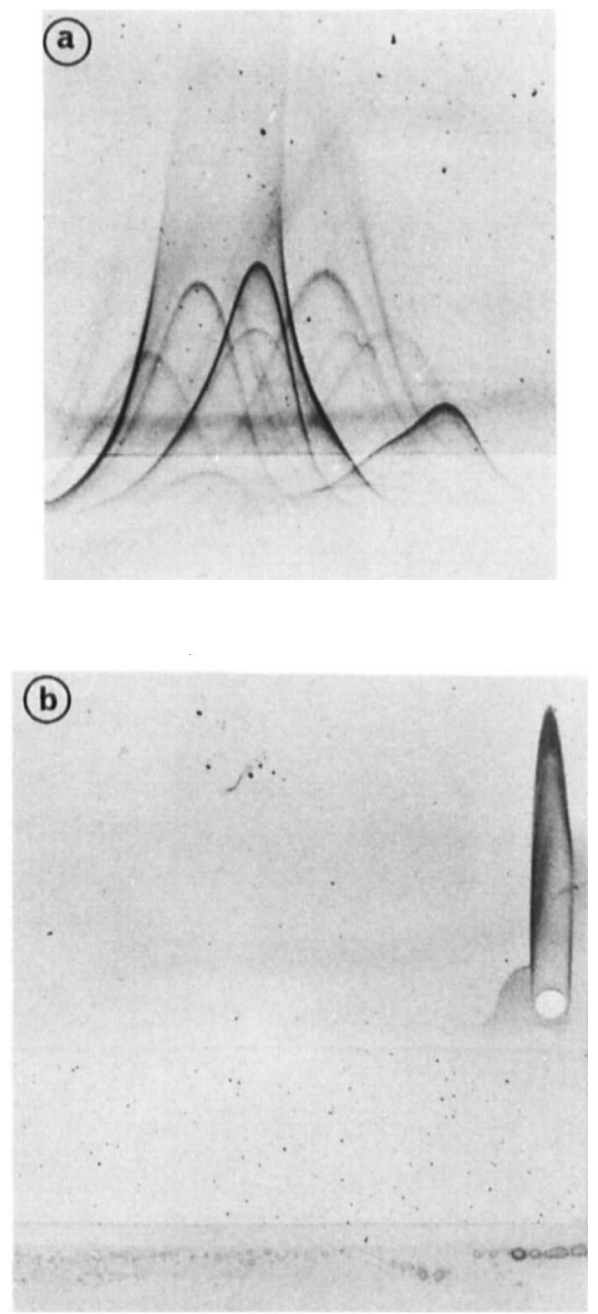

FIG. 9.-Intermediate-gel IEP to determine the effect of pronase on the precipitin pattern when mycelial extracts were tested against test sera. (a) The WS fraction from $A$. fumigatus strain 2109 with serum from an individual patient with aspergilloma; (b) with pronase in the intermediate gel, and an albumin/anti-albumin system in the upper gel. 
precipitin arcs on immunoelectrophoresis when Aspergillus fumigatus preparations were tested with sera from patients with aspergillosis has been reported; of these, the so-called arc $\mathrm{C}$ was detectable with all sera tested while arc $\mathrm{J}$ was identified in $96 \%$ of the cases (Tran Van Ky, Biguet and Fruit, 1966).

Many workers have reported the preparation of wall-located structures with immunological activity. Azuma, Kimura and Yamamura (1968) described the purification and characterisation of immunologically active glycoprotein obtained by pyridine extraction from the defatted mycelium of $A$. fumigatus. Reiss, Stone and Hasenclever (1974b) reported the preparation of a peptido-glucomannan fraction from Candida albicans cell walls which elicited delayed hypersensitivity reactions. Reiss et al. (1974a) prepared a galactomannan-protein complex from $H$. capsulatum with cellular immune activity. Domer (1976) also prepared a glycoprotein antigen from the same organism and compared it with the soluble cytoplasmic substances in tests for cellular immunity. The cytoplasmic material appeared to be the more effective antigen but the relative specificity of these cellular components was not determined.

Whether the wall antigens of A. fumigatus described here have any increased specificity is not yet known. It could be postulated that a glycoprotein structure has enhanced specificity (cf. Pine et al., 1966) in comparison with polysaccharide moieties especially galactomannans, widely distributed in fungi and a cause of much cross-reactivity (Suzuki et al., 1967). Though these preparations consist of a heterogenous mixture of antigens the presence, on 2D IEP, of a limited number of recurring systems that override batch and strain variability could be important in a study of pathogenicity and in the induction of an immune response.

This work was supported by a grant from the Medical Research Council. We thank Mrs E. Wilson and Miss M. Angeli for their excellent technical assistance.

\section{REFERENCES}

AXelsen, N. H., Bock, E. AND KRøll, J. 1973. Comparison of antisera. Scand. J. Immunol., 2, suppl. 1, 101.

Azuma, I., Kimura, H., Hirao, F., Tsubura, E. and Yamamura, Y. 1967. Skin-testing and precipitation antigens from Aspergillus fumigatus for diagnosis of aspergillosis. Am. Rev. resp. Dis., 95, 305.

Azuma, I., Kimura, H. And Yamamura, Y. 1968. Purification and characterization of an immunologically active glycoprotein from Aspergillus fumigatus. J. Bact., 96, 272.

Bardana, E. J., McClatchy, J. K., FARR, R. S. AND Minden, R 1972. The primary interaction of antibody to components of aspergilli. I. Immunologic and chemical characteristics of a non precipitating antigen. J. Allergy clin. Immunol., 50, 208.

Biguet, J., Tran Van Ky, P., Fruit, J. and Andrieu, S. 1967. Identification d'une activité chymotrypsique au niveau de fractions remarquables de l'extrait antigènique d'Aspergillus fumigatus. Repercussions sur le diagnostic immunologique de l'aspergillose. Rev. Immunol., Paris, 31, 317.

Bøg-Hansen, T. C., Brogren, C.-H. AND MCMurrough, J. 1974. Identification of enzymes as glycoproteins containing glucose or mannose. Interaction of barley malt proteins with concanavalin A in electrophoresis. J. Inst. Brew., 80, 443. 
Bøg-Hansen, T. C., Bjerrum, O. J. and Ramlau, J. 1975. Detection of biospecific interaction during the first dimension electrophoresis in crossed immunoelectrophoresis. Scand. $J$. Immunol., 4, suppl. 2, 141.

DOMER, J. E. 1976. In vivo and in vitro cellular responses to cytoplasmic and cell wall antigens of Histoplasma capsulatum in artificially immunized or infected guinea pigs. Infect. Immun., 13,790 .

Dubois, M., Gilles, K. A., Hamilton, J. K., Rebers, P. A. And Smith, F. 1956. Colorimetric method for determination of sugars and related substances. Analyt. Chem., 28, 350.

FrasCH, C. E. 1976. In Dialog no. 13, Removal of detergent by ultrafiltration. Amicon B.V.: Oosterhaut.

HeARn, V. M. AND MACKENZIE, D. W. R. 1979. The preparation and chemical composition of fractions from Aspergillus fumigatus wall and protoplasts possessing antigenic activity. $J$. gen. Microbiol., 112, 35.

HEARN, V. M. AND MACKENZIE, D. W. R. 1980. Antigenic activity of sub-cellular fractions of Aspergillus fumigatus. Zentbl. Bakt. ParasitKde, suppl. 8, 173.

Hearn, V. M., Proctor, A. G. ANd Mackenzie, D. W. R. 1980a. The preparation and partial characterization of antigenic fractions obtained from the mycelial walls of several Aspergillus species. J. gen. Microbiol., 119, 41.

Hearn, V. M., Wilson, E. V., Proctor, A. G. ANd MackenZie, D. W. R. 1980b. Preparation of Aspergillus fumigatus antigens and their analysis by two-dimensional immunoelectrophoresis. J. med. Microbiol., 13, 451.

HollowaY, P. W. 1973. A simple procedure for removal of Triton X-100 from protein samples. Analyt. Biochem., 53, 304.

Huppert, M., Spratt, N. S., Vukovich, K. R., Sun, S. H. and Rice, E. H. 1978. Antigenic analysis of coccidiodin and spherulin determined by two-dimensional immunoelectrophoresis. Infect. Immun., 20, 541.

Kim, S. J., Chaparas, S. D., Brown, T. M. AND Anderson, M. C. 1978. Characterization of antigens from Aspergillus fumigatus. II. Fractionation and electrophoretic, immunologic and biologic activity. Am. Rev. resp. Dis., 118, 553.

LoNGBOTTOM, J. L. 1978. Immunological aspects of infection and allergy due to Aspergillus species. Mykosen, suppl. 1, 207.

Lowry, O. H., Rosebrough, N. J., FarR, A. L. ANd Randall, R. J. 1951. Protein measurement with the folin phenol reagent. J. biol. Chem., 193, 265.

Pine, L., Boone, C. J. AND MCLaughuin, D. 1966. Antigenic properties of the cell wall and other fractions of the yeast form of Histoplasma capsulatum. J. Bact., 91, 2158.

Reiss, E., Mitchell, W. O., Stone, S. H. and Hasenclever, H. F. 1974a. Cellular immune activity of galactomannan-protein complex from mycelia of Histoplasma capsulatum. Infect. Immun., 10, 802.

Reiss, E., Stone, S. H. ANd Hasenclever, H. F. 1974b. Serological and cellular immune activity of peptidoglucomannan fractions of Candida albicans cell walls. Infect. Immun., 9, 881 .

Salvin, S. B. AND RIBI, E. 1955. Antigens from yeast phase of Histoplasma capsulatum. II. Immunologic properties of protoplasm vs. cell walls. Proc. soc. expt. Biol. Med., 90, 287.

SteinbuCh, M. AND AUDRAN, R. 1969. The isolation of IgG from mammalian sera with the aid of caprylic acid. Archs Biochem. Biophys., 134, 279.

Stoddart, R. W. ANd Herber tson, B. M. 1978. The use of fluorescein-labelled lectins in the detection and identification of fungi pathogenic for man: a preliminary study. J. med Microbiol., 11, 315.

Suzuki, S., Suzuki, M., Yokota, K., Sunayama, H. and Sakaguchi, O. 1967. On the immunochemical and biochemical studies of fungi. XI. Cross-reaction of the polysaccharides of Aspergillus fumigatus, Candida albicans, Saccharomyces cerevisiae and Trichophyton rubrum against Candida albicans and Saccharomyces cerevisiae antisera. Jap. J. Microbiol., $11,269$.

Tran Van Ky, P., Biguet, J. And Fruit, J. 1966. Localisation et fréquence des arcs des immunoélectrophorégrammes produits par le sérum des malades atteints de mycétomes aspergillaires appliqués contre l'antigène Aspergillus fumigatus. Rev. Immunol, Paris, 30 , 13. 
WADA, I. 1960. Immunological studies on aspergillosis. I. Immunological reactions in experimental aspergillosis by the filtrate from ground mycelia of Aspergillus fumigatus. Jap.J. Bact., 15, 528.

Yokota, K., Shimada, H., Kamaguchi, A. and Sakaguchi, O. 1977. Studies on the toxin of Aspergillus fumigatus. VII. Purification and some properties of hemolytic toxin (asp-hemolysin) from culture filtrates and mycelia. Microbiol. Immunol., 21, 11. 